\title{
Paraplegia
}

\section{Impairment, Disability, Handicap and Medical Expenses of Persons Aging with Spinal Cord Injury}

\author{
R. R. Menter, MD, G. G. Whiteneck, PhD, S. W. Charlifue, MA, K. Gerhart, \\ RPT, MS, S. J. Solnick, ART, C. A. Brooks, MA, L. Hughes \\ Craig Hospital, 3425 South Clarkson Street, Englewood, Colorado 80110, USA.
}

\begin{abstract}
Summary
Data from 205 Craig Hospital patients with spinal cord injuries (SCI) were analysed with regard to level of injury, age, length of time since SCI, disability (as measured by the Functional Independence Measure), handicap (as measured by the Craig Handicap Assessment and Reporting Technique), and average annual costs for services relating to the SCI. Differences in disability, handicap, and costs of care were analysed by chronological age and length of time since injury. Older individuals showed significant increases in disability and handicap. When chronological age was added to the number of years post-injury, significant increases in disability, handicap, and costs of care were noted at all neurological levels.
\end{abstract}

Key words: Traumatic spinal cord injury; Disability evaluation; Aging; Costs and cost analysis.

As persons with spinal cord injuries (SCI) grow older, additional losses of function can be predicted to occur as the result of normal aging. It has been suggested that the interaction of normal aging processes, when superimposed upon residuals of SCI may lead to a premature decline in functional activities at an earlier point in life than that of able-bodied individuals of similar ages. ${ }^{1}$

To better uderstand the interaction of aging and SCI, and to try to quantify those relationships, the World Health Organization model of disablement was utilised. ${ }^{2}$ This model has divided disablement into three components:

1. Impairment

2. Disability

3. Handicap

\section{Impairment}

Occurs at the organ level and represents 'any loss or abnormality of psychological, physiological, or anatomical structure or function. ${ }^{2}$ In SCI impairment is measured by tools such as manual muscle testing and sensory testing to establish the level and extent of injury. ${ }^{3,4,5}$ 


\section{Disability}

According to the World Health Organization, disability occurs at the person level and is 'any restriction or lack (resulting from an impairment) of ability to perform an activity in the manner or within the range considered normal for a human being. ${ }^{2}$ The extent of disability may be measured by the individual's ability to perform various activities of daily living as in the Barthel Index and the PULSES. ${ }^{6,7}$ Another widely used test for measuring disability, which was chosen for this study, is the Functional Independence Measure (FIM) ${ }^{8}$ Demonstrated to be both valid and reliable, the FIM is a scale which measures the degree of independence for a variety of behaviours including self-care activities, sphincter control, mobility, locomotion, communication, and social cognition. Each of 18 items is rated on a 7-point scale with a score of ' 1 ' representing complete dependence and a score of ' 7 ' signifying complete independence. Intermediate scores document the need for maximal, moderate, or minimal assistance, supervision, and assistive devices.

\section{Handicap}

This occurs at the societal level and has been defined as 'a disadvantage for a given individual resulting from an impairment or a disability that limits or prevents the fulfilment of a role that is normal (depending on age, sex, and social and cultural factors) for that individual.' 2

Until recently, there has been no useful measurement of handicap available to clinicians. Therefore, the Craig Handicap Assessment and Reporting Technique (CHART) was developed to measure this variable. ${ }^{9}$ The CHART is designed to provide a simple, objective scale of the degree to which impairment and disability result in handicaps in the years following initial rehabilitation. Five of the dimensions of handicap described by the World Health Organization ${ }^{2}$ are measured by the CHART:

1. Physical independence

2. Mobility

3. Roles and activities

4. Social integration

5. Economic self-sufficiency

Each dimension is scored on a 100-point scale, utilising items that focus on objectively observable behaviours rather than perceptions or attitudes. All lend themselves to easy quantification. For example, the mobility dimension represents an individual's ability to move about effectively within his or her surroundings and is measured by the hours out of bed per day, days out of the house per week, nights spent away from home per year, home accessibility, and transportation utilised.

The purpose of this study was to investigate differences between individuals with various neurological levels of injury, chronological ages, and durations of injury, to determine if their degrees of disability (FIM score) and handicap (CHART score) differed. In addition, data relating to average annual expenses were gathered to examine variations in medical expenditures during the process of aging. 


\section{Methods}

Medical records were reviewed and telephone interviews were conducted with 205 former clients with (SCI) at the Rocky Mountain Regional Spinal Injury System (Craig Hospital, Englewood, Colorado). Information was gathered relating to degree of impairment (level of injury), functional independence (measured by the FIM), assessment of handicap (measured by the CHART), and medical expenses for the previous year. To determine the latter, names of all providers utilised during the past year were obtained; each was contacted and an itemised bill was obtained. Providers included not only physicians and hospitals, but pharmacists, equipment vendors, and personal care attendants. The study subjects represented four groups of individuals with complete neurological lesions (no distal motor or sensory preservation; ie, Frankel Classification $\mathrm{A}^{10}$ ). Those groups were: $\mathrm{C}-4$ $(n=56), C-6(n=51), T 3-4(n=54)$, and T-11 $(n=44)$. These particular levels were selected to be representative of high and low quadriplegia and high and low paraplegia.

Two-way analyses of variance were performed to determine differences in FIM scores. CHART scores, and annual follow-up expenses by level of injury and by aging. Three approaches were used to look for aging differences. The first approach analysed outcomes for three age groups: 14 to 29 years $(n=58) ; 30$ to 45 years $(n=106)$; and over 45 years $(n=41)$. The second analysis approach attempted to utilise two groupings based on years post-injury; but because only 31 cases were more than 15 years post-injury, this analysis is not reported. Finally, outcomes were analysed by a summation of the two aging factors: chronological age and years post-injury. Two groups were created: those whose chronological age plus years post-injury totalled less than 50 years $(n=134)$, and those whose chronological age plus years post-injury totalled 50 years or more $(n=71)$.

\section{Results}

Table I presents the analysis of disability outcomes (FIM scores). As expected, significant differences among injury levels were found. Those individuals with higher injury levels consistently were more disabled than those with lower injury

Table I Means (and standard deviations) of disability (FIM scores)

\begin{tabular}{lcccc}
\hline & \multicolumn{4}{c}{ By injury level } \\
& C-4 & C-6 & T3-4 & T-11 \\
By age: & & & & \\
$14-29$ years & $50(5)$ & $87(15)$ & $116(2)$ & $116(3)$ \\
$30-45$ years & $51(4)$ & $81(16)$ & $115(3)$ & $116(3)$ \\
$46+$ years & $47(2)$ & $70(13)$ & $108(11)$ & $114(5)$ \\
By age plus years post-injury: & & & & \\
$<50$ years & $50(4)$ & $85(17)$ & $115(3)$ & $115(3)$ \\
$>50$ years & $49(4)$ & $74(11)$ & $111(9)$ & $115(5)$ \\
\hline
\end{tabular}

Statistical significance: In the two-way analysis of variance by injury level and age, both main effects were significant at the $\mathrm{p}<\cdot 001$ level.

In the two-way analysis of variance by injury level and age plus years post-injury, the main effects of injury level and age plus years post-injury were significant at the $\mathrm{p}<\cdot 001$ and $\mathrm{p}<\cdot 01$ levels respectively. 
levels. There also were statistically significant differences in the FIM score analysis by age, with older patients being more disabled than younger patients. Finally, differences obtained when the combination of age plus years post-injury was analysed, also were statistically significant. Individuals whose age plus years postinjury was greater than 50 years were more disabled than the group whose age plus years post-injury was less than 50 years. The greatest differences were found in the C-6 injury level.

Table II presents the results of analysing total CHART scores (handicap outcomes). Both analyses of CHART scores show significant effects of injury level, with higher level injuries being more handicapped. Significant differences also were found when CHART scores were analysed by age, with a clear pattern of greater handicap among older SCI individuals. Similarly, there were significant differences in the analysis of age plus years post-injury. Individuals whose age plus years post-injury totalled more than 50 years were more handicapped than those whose age plus years post-injury totalled less than 50 years.

Further analysis of the previously described five dimensions of CHART by age plus years post-injury revealed significant differences in four of the five subscales. Individuals whose age plus years post-injury totalled to more than 50 years demonstrated patterns of less physical independence, less mobility, more restricted roles and activities, and less social integration than their younger counterparts. No significant differences were found on the measure of economic self-sufficiency.

Table III presents the results of analysing annual follow-up costs. Again, significant differences were found by injury level with higher levels of injury incurring greater follow-up medical expenses. No significant differences were found when age alone was the independent variable. A statistically significant difference was found, however, in the analysis of age plus years post-injury. Substantially greater costs were found for patients whose age plus years post-injury totalled more than 50 years. The magnitude of the average cost difference between the two groups whose age plus years post-injury was over or under 50 years was substantial. The costs of the aging group ranged from one third greater for those with $\mathrm{C}-6$ injuries, to two thirds greater for those with $\mathrm{C}-4$ injuries, to approximately double for those with paraplegia. Subsequent analysis revealed larger hospital and physician expenses for the aging group across all four injury

Table II Means (and standard deviations) of handicap (CHART scores)

\begin{tabular}{lcccc}
\hline & \multicolumn{5}{c}{ By injury level } \\
& & $\mathrm{C}-6$ & $\mathrm{~T}-4$ & $\mathrm{~T}-11$ \\
By age: & $359(74)$ & $436(63)$ & $481(34)$ & $462(33)$ \\
$14-29$ years & $364(104)$ & $398(84)$ & $445(67)$ & $446(65)$ \\
$30-45$ years & $216(104)$ & $363(42)$ & $399(68)$ & $437(43)$ \\
$46+$ years & & & & \\
By age plus years post-injury: & $372(98)$ & $426(64)$ & $447(67)$ & $467(30)$ \\
$<50$ years & $277(95)$ & $359(82)$ & $433(68)$ & $426(63)$ \\
$>50$ years & & & \\
\hline
\end{tabular}

Statistical significance: In the two-way analysis of variance by injury level and age, the main effects of injury level and age was significant at the $\mathrm{p}<\cdot 001$ and $\mathrm{p}<\cdot 01$ levels respectively.

In the two-way analysis of variance by injury level and age plus years post-injury, both main effects were significant at the $\mathrm{p}<\cdot 001$ level. 
Table III Means (and standard deviations) of annual follow-up costs

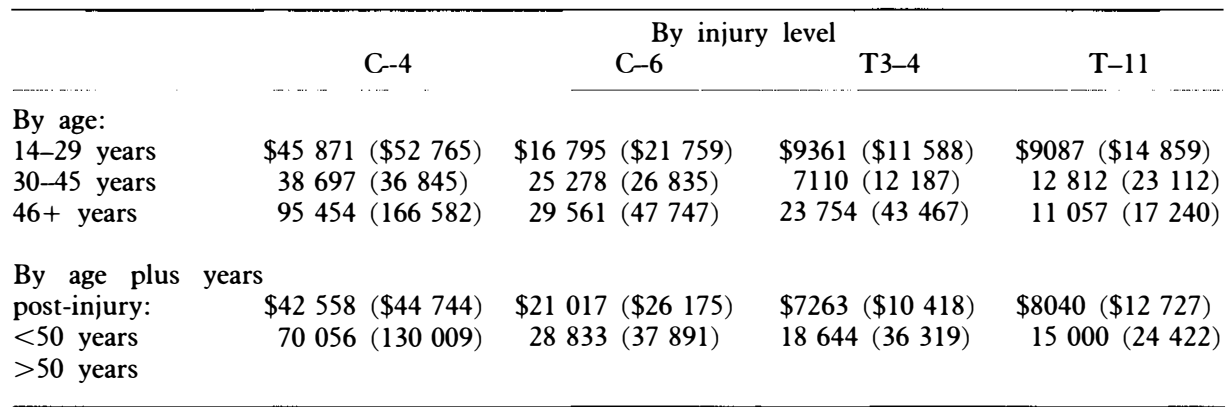

Statistical significance: In the two-way analysis of variance by injury level and age, both main effect of injury level was significant at the $\mathrm{p}<\cdot 001$ level. The main effect of age was not significant.

In the two-way analysis of variance by injury level and age plus years post-injury, the main effects of injury level and age plus years post-injury were significant at the $\mathrm{p}<\cdot 001$ and $\mathrm{p}<.05$ levels respectively.

levels. Substantially greater attendant care expenses were also found for the aging group of $\mathrm{C}-4$ quadriplegics. These findings were obtained despite the fact that there were extremely large standard deviations indicating great individual variations and skewed distributions.

\section{Discussion}

The present study suggests that the process of aging with SCI will be accompanied with increased disability, increased handicap, and increased costs of care. Significant differences in all three of these outcomes were found to be associated with a combination of chronological age and years post-injury. This may indicate that the best conceptual model of aging with SCI is an additive model that includes both factors.

Chronological age alone was significantly related to increased disability and handicap. While these declines in physical and psychosocial functioning might be expected to occur in older able-bodied individuals, they appear to occur much earlier in the SCI population.

The Functional Independence Measure, used for this study, bases its scores on the activities an individual actually does perform as opposed to what the individual can perform. While statistically significant differences in disability (FIM scores) were found, the magnitude of the differences were relatively small except for the C-6 injury level group. Subjective observations of the study participants have led the authors to the conclusion that functional declines may be greater than measured. Specifically, during interviews with study participants, several individuals indicated that while they remained independent in a number of their activities of daily living and still completed tasks in what could be considered a reasonable amount of time (thereby receiving higher scores), they now took more time and effort to complete the same activities. Future studies need to examine the hypothesis that greater effort is required to maintain independence as aging occurs and more sophisticated measures of disability may be required.

The data relating to handicap as measured by the CHART clearly indicate general declines with aging. Statistically significant declines were found in older individuals and in individuals whose age plus years post-injury totalled to 50 or 
more years. There were changes in four of the five CHART dimensions related to aging: physical independence, mobility, roles and activities, and social integration. As scores in these dimensions decline, a pattern of evolving needs in all areas of life activity can be expected. Health care providers therefore need to be aware of the changing physical, psychosocial, and socioeconomic needs of individuals aging with spinal cord injuries. New follow-up techniques should be employed to provide early intervention rather than crisis management. Clinicians need to be aware of the potential declines their clients may experience and must be wellversed in developing and offering compensating resources and lifestyle strategies that will allow individuals to maintain the quality of life they had enjoyed in the early years after their injuries. Clearly, at all levels of injury and regardless of duration, 'success' will need to be measured not only in terms of scores attained or by physical independence alone, but by satisfaction with the quality of life. This will be crucial in determining and justifying the individual's changing needs for support, personal assistive care, and equipment and adaptations.

Perceived quality of life following SCI has not been addressed specifically in this study. Despite the presence of functional declines that are probably associated with aging, many individuals report an increasing satisfaction with life. ${ }^{11}$ It is imperative, therefore, that attention be focused on individuals' functional declines in a manner that maintains or improves perceived life satisfaction. The aforementioned and future studies focusing on the identification and measurement of quality of life issues will be a crucial component in the continued study of aging and spinal cord injury. Programmes designed to enhance the lives of individuals who are newly injured, as well as those who are aging with SCI, are the ultimate goal.

The increasing cost of medical care is a concern to health care providers, consumers, and policy planners. This study demonstrates that annual follow-up costs for individuals with SCI increase inconsistently with age and increase dramatically when age and years post-injury are considered in combination (Table III). Increased physician and hospital costs reflect greater utilisation of services due to medical complications that accompany increasing age and length of time postinjury. Increased attendant care expenses may reflect greater dependency. Health planners need to consider long term changes in health care expenditures in order to design programmes which can continue to meet the costs of care in later years.

Much more work needs to be done on a larger study population to answer the following questions: Are specific neurological levels such as C-6 and T-3 more vulnerable to the aging process because they must work harder to maintain their levels of function? Are there specific characteristics which exaggerate or magnify the effects of aging such as obesity or smoking. Do certain lifestyles, habits, and/or therapy approaches act to forestall the effects of aging?

SCI care has made great strides in acute care and early rehabilitation management. With a better understanding of the effects of aging, it will be possible to develop the necessary support systems to ensure a high quality of life for people aging with SCI.

\section{Acknowledgement}

This research was supported by a National Institute of Disability and Rehabilitation Research (NIDRR) grant, G008535132. 


\section{References}

1. Ohry A, Shemesh Y, Rozin R. Are chronic spinal cord injured persons prone to premature aging? Medical Hypotheses 1983:11:467-469.

2. World Health Organization. 1980 International Classification of Impairments Disabilities and Handicaps. Geneva.

3. Bracken MB, Shepard MJ, Collins WF et al. A randomized, controlled trial of methylprednisolone or naloxone in the treatment of acute spinal-cord injury. The New England Fournal of Medicine 1990:322:20:1405-1411.

4. Daniels L, Worthingham C. 1972. Muscle Testing: Techniques of Manual Examination. 3rd edn. WB Saunders Co, Philadelphia, Pennsylvania.

5. Kendall HO, Kendall FP, Wadsworth GE. 1971. Muscles: Testing and Function. 2nd edn. Williams and Wilkins Co., Baltimore, Maryland.

6. Mahoney FT, Barthel DW. Functional evaluation: The barthel index. Maryland State Medical Fournal 1965:14:61-65.

7. Moskowitz E, McCann CB. Classifications of disability in the chronically ill and aging. Fournal of Chronic Diseases 1957:5:342-346.

8. Hamilton BB, Granger CV, Sherwin FS, Zielezny M, Tashman JS. A uniform data set for medical rehabilitation. In: Fuhrer MJ, ed. Rehabilitation Outcomes. Paul H. Brookes Publishing Co., Baltimore, Maryland, 1987.

9. Whiteneck GG, Charlifue SW, Gerhart KA, Overholser JD, Richardson GN. The Craig Handicap Assessment and Reporting Technique (CHART), Craig Hospital, Englewood, Colorado, 1987.

10. American Spinal Injury Association. Standards for Neurological Classification of Spinal Injury Patients. Chicago. 1982: p. 9-10.

11. Creek G, Moore M, Oliver M, Salisbury V, Silver J, Zarb G. 1987. Personal and Social Implications of Spinal Cord Injury: A Retrospective Study. Thames Polytechnic, London. 